\title{
On the ability to inhibit complex thoughts: A stop-signal study of arithmetic
}

\author{
GORDON D. LOGAN \\ Purdue University, West Lafayette, Indiana \\ and \\ CAROL Y. BARBER \\ University of Guelph, Guelph, Ontario, Canada
}

\begin{abstract}
An experiment was conducted to determine whether the stop-signal procedure of Logan (1983, 1985) is sensitive enough to detect the presence of inhibited thoughts when thoughts are in fact inhibited, and to determine whether the stop-signal procedure is susceptible to Zeigarnik-type effects. Subjects added several columns of nine digits and were interrupted occasionally. A subsequent recognition memory test showed that interrupted problems were remembered less accurately than completed problems and that memory for interrupted problems was worse the earlier the interruption occurred. These results suggest that the stop-signal procedure is sensitive enough to detect thoughts that actually were inhibited and that the stop-signal paradigm is not susceptible to Zeigarnik-type effects even when it is applied to complex tasks.
\end{abstract}

Logan (1983) investigated the ability to inhibit thought by asking subjects to inhibit the overt actions associated with thought and then examining the aftereffects of thought to see whether the thoughts were inhibited with the actions. Specifically, he asked subjects to make category or rhyme judgments about pairs of words and had them inhibit the responses associated with the judgments about $40 \%$ of the time. Later, he asked them to recognize word pairs whose responses were and were not inhibited. He found that memory for word pairs whose responses were inhibited was about the same as memory for word pairs whose responses were not inhibited, and memory for word pairs whose responses were inhibited was equal for responses that were inhibited early (100 msec after stimulus onset) and responses that were inhibited late (700 msec after stimulus onset). These results led Logan to conclude that the thoughts underlying category and rhyme judgments were not inhibited with the overt responses, but rather, they ran to completion ballistically. Later, he corroborated these conclusions using repetition priming as the index of thought (Logan, 1985).

It may be the case, however, that Logan's procedure was not sensitive enough to discriminate thoughts that were inhibited from thoughts that were not inhibited. Possibly, memory performance does not depend on whether or not the underlying thoughts are completed. Indeed,

This research was supported by Grant U0035 to Gordon Logan from the Natural Sciences and Engineering Research Council of Canada. We would like to thank Jane Zbrodoff, Henry Roediger, and Richard Schweikert for comments on the article. Requests for reprints should be addressed to G. D. Logan, Department of Psychological Sciences, Purdue University, West Lafayette, IN 47907.
Zeigarnik (1927) and others found that complex thoughts were better remembered if they were interrupted than if they were completed without interruption.

The purpose of the present experiment was to determine whether Logan's stop-signal procedure discriminates between thoughts that are interrupted and thoughts that are completed, and to determine whether Zeigarnik-type effects (i.e., better memory for interrupted than completed thoughts) occur in the stop-signal paradigm. Zeigarnik's procedure differed from Logan's in several respects, each of which may be responsible for the difference in results. First, Zeigarnik's subjects performed several complex tasks, such as unscrambling scrambled sentences, canceling vowels on a page of text, and making as many words as possible out of a limited set of letters. By contrast, Logan's subjects performed two relatively simple tasks: deciding whether two words rhymed and deciding whether one word belonged to the category named by the other. Possibly, complex tasks and simple tasks are governed by different principles. Second, Zeigarnik tested memory for the tasks themselves, whereas Logan tested memory for the stimuli presented during each task. Caron and Wallach (1957) had subjects unscramble several scrambled sentences and tested for recognition of the sentences themselves. They found no difference between completed and uncompleted tasks. Third, Zeigarnik used recall to test memory, whereas Logan used recognition. Pachauri (1935) found that subjects in Zeigarnik's procedure tended to recall the interrupted tasks first, so memory for completed tasks may have been depressed by output interference. The recognition test used by Logan (1983) and by Caron and Wallach (1957) would not be susceptible to such strategic factors, since "output order" was controlled by the experimenter. 
To assess the importance of these factors, an experiment was conducted in which subjects performed just one task for several trials, being interrupted on occasion, and subsequently were tested for recognition memory for the materials. Specifically, subjects added columns of nine digits, and on one third of the trials, they were stopped after they added the second, fourth, sixth, or eighth digit. Then they performed a recognition test in which half of the problems were old and half were new. Of the old problems, half had been interrupted and half had not been interrupted. Subjects were required to add the nine digits in each problem and then say whether or not they recognized that problem. If the stop-signal paradigm is sensitive enough to detect the inhibition of thought, then memory for the interrupted problems should be worse than memory for the completed problems, and memory for interrupted problems should be worse for those problems that were interrupted earliest. However, if the stop-signal paradigm is not sensitive enough to detect the inhibition of thought, then memory for problems that were interrupted should be no different from memory for problems that were not interrupted, and memory for problems that were interrupted should be the same whether the interruption occurred early or late in the problem. Finally, if the stop-signal paradigm is susceptible to Zeigarnik-type effects, then memory for interrupted problems should be better than memory for uninterrupted problems. Zeigarnik's (1927) interpretation of interruption does not make predictions about the effects of stop-signal delay.

\section{METHOD}

\section{Subjects}

The subjects were 32 undergraduate students who served for course credit or monetary compensation. All were naive as to the purpose of the experiment.

\section{Apparatus and Stimuli \\ The stimuli were nine-digit addition problems, constructed by sam- pling with replacement the digits one through nine. A different set of problems was constructed for each subject by a DEC-10 computer, which printed them in adjacent columns on standard $30 \times 38 \mathrm{~cm}$ computer paper. Adjacent columns were separated by eight space characters. \\ The stop signal was the word "stop" spoken by the experimenter. Stop signals, when presented, occurred after the subjects spoke the sum, following addition of the second, fourth, sixth, or eighth digit.}

\section{Procedure}

Each subject completed two blocks of trials, one for the orienting task and one for the recognition task. The orienting task consisted of 20 trials. On each trial, subjects added aloud a column of nine digits and reported the sum. Stop signals occurred on 8 of the trials, twice at each delay (i.e., after adding the second, fourth, sixth, and eighth digit). The order of stop-signal versus no-signal trials and the order of stop-signal delays were randomized separately for each subject.

The recognition block consisted of 32 trials. Half of the trials involved problems that had been presented in the orienting task (old problems) and the other half involved problems that had not been presented in the orienting task (new problems). The old problems consisted of the 8 problems that had been presented with a stop signal and 8 of the 12 problems that had been presented without a stop signal. Old and new problems were mixed randomly, and a separate random order was prepared for each subject.
Subjects were instructed before each block. In the orienting block, they were told to add each column from top to bottom as quickly and accurately as possible, beginning when the experimenter said "go" and ending when the subjects reported the final sum or when the experimenter said "stop." They were told to add the digits aloud, reporting each digit and each partial sum. After they spoke the final sum, they were to write it at the bottom of the column. The entire sheet of problems remained in view at all times. Note that we did not mention the recognition block when we described the orienting task to the subjects; we wanted to measure incidental learning.

In the recognition block, subjects were told to add each column aloud, reporting each digit, each partial sum, and the final sum, and then to decide whether or not they had seen that problem in the orienting task. They were told that half of the problems were old and half were new.

In both blocks, we measured with a stopwatch the time subjects took to add the nine digits (or to reach the appropriate partial sum on stopsignal trials), and we tape-recorded each trial to check the accuracy of our measures.

\section{RESULTS}

\section{Orienting Task}

Subjects stopped immediately after reporting the partial sum on each stop-signal trial. Stop-signal trials therefore represent trials on which the chain of processing underlying an entire nine-digit problem was interrupted. Moreover, subjects' processing was less complete the earlier the stop-signal delay.

The mean time taken to produce the final sum on nosignal trials was $16.48 \mathrm{sec}$. The mean times taken to report the appropriate partial sums on stop-signal trials were .97 , $6.84,9.80$, and $14.79 \mathrm{sec}$ for stop signals that occurred after the second, fourth, sixth, and eighth digit was added, respectively. The latter times increased monotonically with stop-signal delay, reflecting a general finding that the time taken to perform complex arithmetic increases linearly with the number of simple operations (Dansereau \& Gregg, 1966; Marsh \& Maki, 1976; McClain \& Shih Huang, 1982).

On no-signal trials, subjects' final sums were correct $69.3 \%$ of the time. Assuming that the component operations were independent of each other (and therefore taking the eighth root of .693), this corresponds to an accuracy of $95.5 \%$ for each simple addition operation.

\section{Recognition Task}

The mean hit rates for stop-signal trials are presented as a function of stop-signal delay in Figure 1, along with the mean hit rate from no-signal trials and the mean false alarm rate. Memory performance was worse on stopsignal trials than on no-signal trials, and it improved, as stop-signal delay increased, from near-chance levels at the shortest delay to a level near no-signal performance at the longest delay.

These results were confirmed by ANOVA on the hit rates. The main effect of the five conditions (i.e., the four stop-signal conditions and the no-signal condition) was significant $[\mathrm{F}(4,124)=3.51, \mathrm{p}<.01, \mathrm{MSe}=.1135]$, as was a contrast comparing the no-signal condition with the mean of the four stop-signal conditions $[F(1,124)=$ $8.61, \mathrm{p}<.01]$, and a trend test evaluating the linear in- 


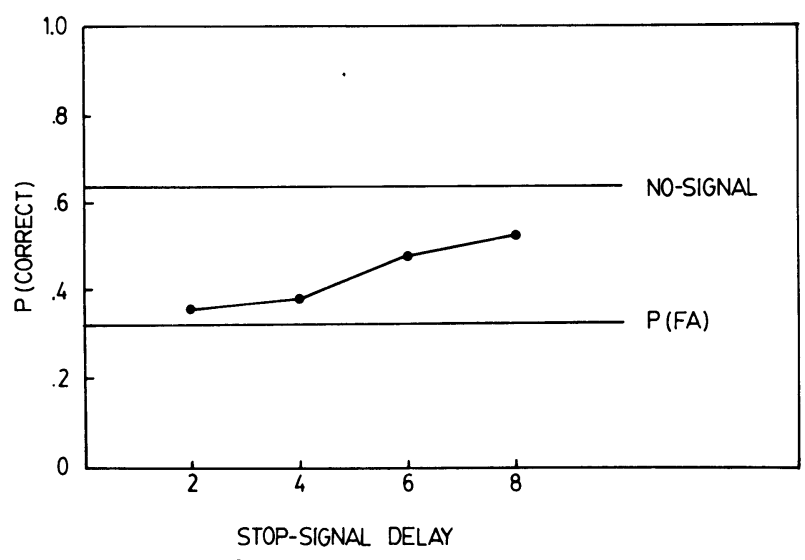

Figure 1. Proportion of correct responses (hits) in the memory task as a function of stop-signal delay. Proportion correct from trials on which no stop signal was presented (horizontal line marked NOSIGNAL) and the proportion of false alarms [horizontal line marked P(FA)] are also plotted.

crease in hit rate with delay in the stop-signal conditions $[\mathrm{F}(1,124)=5.24, \mathrm{p}<.05]$.

\section{DISCUSSION}

The orienting data suggest that subjects stopped processing the addition problems as soon as the stop signal was presented. Moreover, they stopped processing sooner the earlier the stop signal was presented. The recognition data showed that memory was poorer on stop-signal trials than on no-signal trials, and that memory performance improved as stopsignal delay increased on stop-signal trials. This pattern of results suggests that the underlying processes were inhibited with the overt responses. Thus, the stop-signal paradigm appears to be sensitive enough to indicate that underlying thought processes were interrupted when in fact they were interrupted.

These results suggest that Logan's $(1983,1985)$ earlier results may not have been an artifact of the insensitivity of the stop-signal paradigm. Thus, the stop-signal procedure appears to be a reasonable paradigm in which to assess the inhibition of thought as well as action. The results also indicate that a Zeigarnik effect need not occur when complex thoughts are interrupted, at least if subjects are asked to recognize the stimuli they were thinking about. The Zeigarnik effect may occur only when memory for the tasks themselves is tested (cf. Caron \& Wallach, 1957) or when free recall is used to test memory (Pachauri, 1935).

\section{REFERENCES}

Caron, A. J., \& Wallach, M. A. (1957). Recall of interrupted tasks under stress: A phenomenon of memory or of learning? Journal of Abnormal \& Social Psychology, 55, 372-381.

Dansereau, D. F., \& GREGG, L. W. (1966). An information processing analysis of mental multiplication. Psychonomic Science, 6, 71-72.

LOGAN, G. D. (1983). On the ability to inhibit simple thoughts and actions: I. Stop-signal studies of decision and memory. Journal of Experimental Psychology: Learning, Memory \& Cognition, 9, 585-606.

LOGAN, G. D. (1985). On the ability to inhibit simple thoughts and actions: II. Stop-signal studies of repetition priming. Journal of Experimental Psychology: Learning, Memory \& Cognition, 11, 675-691.

MARSH, L. G., \& MAKI, R. H. (1976). Efficiency of arithmetic operations in bilinguals as a function of language. Memory \& Cognition, 4, 459-464.

McClain, L., \& Shih Huang, J. Y. (1982). Speed of simple arithmetic in bilinguals. Memory \& Cognition, 10, 591-596.

Pachauri, A. R. (1935). A study of Gestalt problems in completed and interrupted tasks: Part 1. British Journal of Psychology, 25, 365-381.

ZEIGARNIK, B. (1927). Über das Behalten von erledigten und unerledigten Handlungen. Psychologische Forschung, 9, 1-85.

(Manuscript received for publication May 21, 1985.) 